\title{
Mobile Ad Hoc Nanonetworks with Collision-based Molecular Communication
}

\author{
Aydin Guney, Baris Atakan, Student Member, IEEE, and Ozgur B. Akan, Senior Member, IEEE
}

\begin{abstract}
Recent developments in nanotechnology have enabled the fabrication of nanomachines with very limited sensing, computation, communication, and action capabilities. The network of communicating nanomachines is envisaged as nanonetworks that are designed to accomplish complex tasks such as drug delivery and health monitoring. For the realization of future nanonetworks, it is essential to develop novel and efficient communication and networking paradigms. In this paper, the first step towards designing a mobile ad hoc molecular nanonetwork (MAMNET) with electrochemical communication is taken. MAMNET consists of mobile nanomachines and infostations that share nanoscale information using electrochemical communication whenever they have a physical contact with each other. In MAMNET, the intermittent connectivity introduced by the mobility of nanomachines and infostations is a critical issue to be addressed. In this paper, an analytical framework that incorporates the effect of mobility into the performance of electrochemical communication among nanomachines is presented. Using the analytical model, numerical analysis for the performance evaluation of MAMNET is obtained. Results reveal that MAMNET achieves adequately high throughput performance to enable frontier nanonetwork applications with acceptable communication latency.
\end{abstract}

Index Terms-Nanomachines, Molecular neuro-spike communication, Mobile ad hoc molecular nanonetworks, Epidemic spreading, Delay and throughput performance.

\section{INTRODUCTION}

Rapid growth in nanotechnology provides favorable development in miniaturization and fabrication of nanomachines with simple sensing, computation, communication, and action capabilities. In literature, three different ways are proposed for the development of nanomachines, namely, bottom-up, top-down and bio-hybrid approaches [1]. In bottom-up approach, the molecules or atoms are assembled to form nanomachines. In top-down approach, the design of nanomachines is realized by downsizing current microelectronic devices. The third approach is the bio-hybrid approach. In this approach, biological entities can either be genetically modified to develop nanomachines or used as the building blocks of nanomachines. In nature, there are already many molecular-scale phenomena consisting of an arranged set of molecules, which are able to perform very simple tasks. These phenomena can be envisaged as naturemade nanomachines. For example, a chloroplast in a plant cell stands as a nanomachine including arrays of molecules that act as tuned optical antennas for absorption and transformation of solar energy. Mitochondrion can be envisioned as a nanomachine used for controlled combustion of organic molecules to generate adenosine triphosphate (ATP) for fulfilling energy needs of cellular activities. A flagellar motor attached on the membrane of many bacterial cells is a highly structured combination of proteins for providing

The authors are with the Next-generation Wireless Communications Laboratory, Department of Electrical and Electronics Engineering, Koc University, Istanbul, 34450, Turkey (e-mail: akan@ku.edu.tr).

This work was supported in part by the Turkish Scientific and Technical Research Council under grant \#109E257, by the Turkish National Academy of Sciences Distinguished Young Scientist Award Program (TUBA-GEBIP), and by IBM through IBM Faculty Award. cellular movement [4]. Nature-made nanomachines can be exploited to learn and understand the principles governing the operation of nanomachines and their interactions [4].

The limited capabilities of nanomachines also limit the applications that can be achieved by a single nanomachine. However, the networks of communicating nanomachines, i.e., nanonetworks, are expected to enable very large set of new applications in various research fields such as genetic engineering, health monitoring, and military surveillance systems [1]. For the realization of frontier nanonetworks, it is imperative to develop new and efficient communication and networking techniques. The aim of these techniques is to overcome unique challenges and requirements of nanonetworks. These challenges and requirements can be briefed as follows:

- Scale of the nanomachines is on the order of micrometers, therefore, classical transceiver circuitries cannot be mounted into nanomachines.

- Current encoding and decoding techniques are not feasible due to very limited processing capability of nanomachines.

- For in-vivo application scenarios, nanomachines need to be biocompatible in order not to be rejected by the organism.

- Mobility of nanomachines is governed by the physical rules in nano domain [11].

- Nanomachines are extremely susceptible to any change in the communication environment such as rapid concentration change or quaking.

- Communication or noise signal characteristics cannot be easily anticipated due to severely unreliable nature of the communication medium. 
In literature, different communication techniques, namely, acoustic, electromagnetic, molecular, and nanomechanical, are proposed for the communication of nanomachines [14]. Traditional electromagnetic and acoustic communication technologies cannot be directly used in the communication between nanomachines because of the size restrictions. Nanomechanical communication requires a strict physical contact between transmitter and receiver in order to enable information transmission, which makes it inconvenient for many application scenarios. On the other hand, molecular communication, which is already used by biological entities, is a promising approach for the communication of nanomachines.

Molecular communication is inspired by the natural nanoscale communication techniques. In living organisms, cells communicate in various ways. Existing communication paradigms between cells may be adopted for the realization of nanonetworks [5]. In [8], a design of a molecular communication system based on intercellular calcium signaling is introduced. In [7] and [10], molecular communication systems using biological molecular motors and vesicles as communication carriers are introduced. Molecular communication channel is modeled as a binary symmetric channel and mutual information and capacity expressions are derived for that channel in [6]. A flagellated bacteria and catalytic nanomotors based molecular nanonetworks is proposed in [3]. In [12], a computational model for mobile nanomachines using molecular communication is introduced. In [2], the first realistic and very comprehensive physical channel model of diffusion-based molecular communication has been developed.

In literature, generally, nanomachines are considered as immobile nodes. However, mobile nanomachines may be indispensable for many nanonetwork applications. A possible example that necessitates the mobile nanomachines is a nanonetwork designed for coordinated cancer cell detection by identifying cancer cells and informing a central controller to take an appropriate action. Clearly, these applications necessitate the realization of mobile ad hoc molecular nanonetworks (MAMNET). In MAMNET, nanomachines collect some environmental information such as a chemical state, or the existence of a certain concentration level and deliver the information collected by mobile nanomachines to mobile infostations as shown in Fig. 1. The infostations are central control units that make decisions according to the collected information, or gateways that connect MAMNET to a micro-device.

However, the mobility of nanomachines also incurs a new set of crucial challenges that must be addressed for the realization of MAMNET. One of the main challenges introduced by the mobility is the intermittent connectivity, i.e., nanomachines can communicate only when they are in physical contact. Therefore, this intermittent connectivity clearly imposes a high level of latency on nanoscale communication. To the best of our knowledge, the feasibility and performance of an ad hoc nanonetwork that is composed of mobile nanomachines communicating through electrochemical means have not yet been investigated.

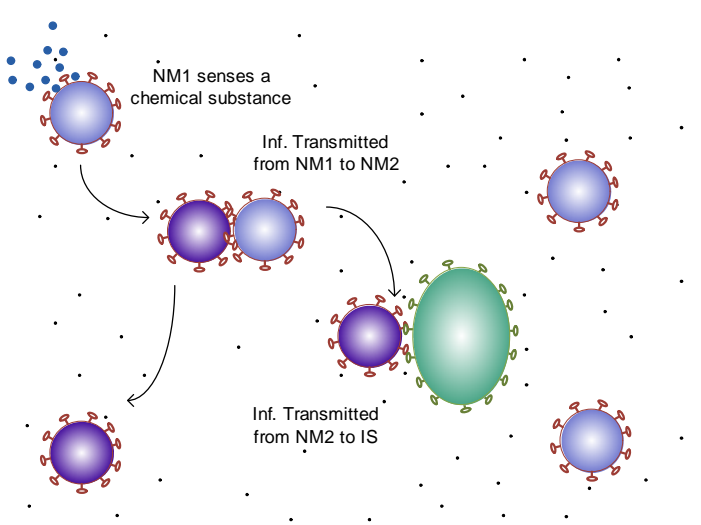

Fig. 1. Information flow in MAMNET.

The aim of this paper is to introduce the concept of MAMNET and provide an analytical framework in order to show feasibility of MAMNET. The communication of nanomachines is inspired by the cellular communication paradigm in immune system. In immune system, the immune response starts by the recognition of antigens by $\mathrm{T}-$ cells. However, T-cells cannot directly recognize antigens. First, antigen presenting cells (APCs) recognize the antigen entering the body. Then, APCs and T-cells collide and adhere to each other, which forms an immunological synapse by the interaction of complex molecules on the surface of APCs with T-cell receptors [13]. This allows the transfer of antigen information to T-cells and the immune response starts.

In MAMNET, the communication of mobile nanomachines is similar to the natural cellular communication in immune system and enabled by three main phases, namely, collision, adhesion and transmission. In collision phase, nanomachines randomly collide with each other. In adhesion phase, nanomachines stuck to each other. Finally, the information is transmitted in the transmission phase. For the transmission of information, we introduce a new communication paradigm, called molecular neurospike communication, that is inspired by electrochemical communication among biological neuron cells. After the adhesion phase, molecular neuro-spike communication scheme enables the communication of adherent nanomachines. These three phases briefly mentioned above jointly provide the communication of nanomachines and infostations in MAMNET.

The remainder of this paper is organized as follows. In Section 2, we give an overview of MAMNET, and underline the design issues and assumptions. Then, we model the collision and adhesion of nanomachines in Section 3. In Section 4, we introduce molecular neuro-spike communication of adherent nanomachines and an analytical capacity expression for molecular neuro-spike channel. In Section 5 , we derive the distribution of communication latency and find average throughput in MAMNET. We present the numerical results in Section 6 and give concluding remarks in Section 7. 


\section{Mobile Ad Hoc Molecular nanonet- WORK COMMUNICATION MODEL}

MAMNET is composed of two kinds of mobile nanonodes, namely, nanomachines and infostations as shown in Fig. 1. Nanomachines and infostations are assumed as genetically modified cells with additional capabilities. Nanomachines are assumed to sense the environment and gather some environmental information to deliver infostations. Based on the collected information coming from nanomachines, infostations are assumed to make decision for an appropriate action. Furthermore, infostations may also be considered as gateways that connect MAMNET to a micro-device.

Nanomachines and infostations do not always have a direct communication interface because of mobility. In order for a nanomachine to transmit collected information to an infostation, the nanomachine and the infostation should first collide, then adhere to each other. Since the system volume containing MAMNET is much larger than the size of a single nanonode, the meeting probability of a nanomachine and an infostation is very low. Thus, intermediary nanomachines can be used as relay nodes that help the communication between the nanomachines and the infostations as follows.

Fig. 1 describes the information flow in MAMNET. Nanomachine 1 is assumed to be the source of the information that is to be communicated to the infostation. Nanomachine 1 transmits the information to every nanomachine, i.e., relay node, with which it collides and adheres. The nanomachines that acquire the information follow the same strategy and this clearly increases the probability of information delivery. Note that this communication strategy is clearly similar to the spreading of epidemic disease.

Here, we assume that nanomachines and infostations do not have any additional capability for mobility or collision. As will be detailed in Section 3, nanomachines diffuse in the environment and randomly collide with each other. The collided nanonodes adhere to each other via the surface mounting molecules called, ligands and receptors. This phenomenon is commonly known as the ligand-receptor binding process. According to this process, ligand molecules on the surface of one biological entity, i.e., nanonode, bind to the receptors of an other nanonode. Once a certain number of bonds is established between the nanonodes, they can adhere to each other.

The performance of MAMNET directly depends on the interaction of nanonodes that are triggered by collision and adhesion of nanomachines and infostation. Therefore, it is important to investigate the collision rate, i.e., the number of collisions occur per unit time, and the adhesion probability of collided nanomachines and infostations. Next, a detailed analytical analysis of collision and adhesion phases in MAMNET is given.

\section{Collision and Adhesion of Nanoma- CHINES}

In nature, the behaviors of cells are significantly affected by contacts with other cells [17]. In their environment,

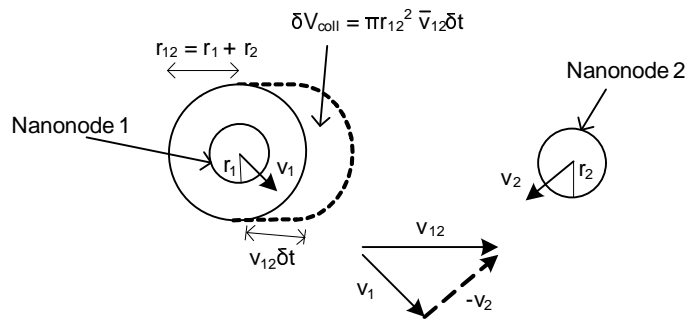

Fig. 2. Collision of two nanomachines. Nanomachine 1 and nanomachine 2 collide in the time interval $\delta t$ if nanomachine 2 is in the collision volume $\delta V_{\text {coll }}$ in $\delta t$.

mobile biological cells collide with each other and cellular adhesion occurs between the collided cells. The cell adhesion is accomplished by the ligand-receptor binding process on the surface of the cells. The adhesion has a critical role in intracellular and intercellular signaling, i.e., communication, that governs basic cellular activities and coordinates cell actions [19].

In this paper, we adopt the natural cellular communication for the communication among nanomachines. Similar to living cells, nanomachines should be in physical contact with each other in order to communicate. The physical contact is established through the collision and adhesion of nanonodes that are modeled as follows.

\subsection{Collision of Nanomachines}

As in traditional mobile ad hoc networks (MANET), in MAMNET stochastic nature of nanomachine collision rate is governed by some mobility models such as brownian motion and random waypoint. Since nanomachines do not have any capability for controlling their movement, they are assumed to freely diffuse in the aqueous medium. Hence, the mobility of nanomachines is governed by the dynamics of Brownian motion that explains the movement of small particles that freely diffuse in an aqueous medium.

Nanomachines with radius $r$ are assumed to be contained in a volume $V$ and moving according to Brownian motion. Here, we assume that $V>>r$. In order to find the collision rate of nanomachines, we first obtain the probability that the nanomachines collide within the next infinitesimal time interval $\delta t$, given that the first nanomachine's center is located at the position $\left(x_{1}, y_{1}, z_{1}\right)$. Two nanomachines collide in the next $\delta t$, only if the second nanomachine is in the volume that is covered by the first nanomachine with respect to the second nanomachine. This collision volume $\delta V_{\text {coll }}$ is shown in Fig. 2 and expressed as

$$
\delta V_{\text {coll }}=\pi r_{12}^{2} v_{12} \delta t
$$

where $r_{12}=r_{1}+r_{2}$ and $v_{12}$ is the relative velocity of the first nanomachine with respect to the second nanomachine. By using the relative velocity, the second nanomachine is considered as stationary while the first nanomachine is considered moving with velocity $v_{12}$ rather than $v_{1}$. Thus, the probability that the second nanomachine, i. e., 
two nanomachines collide, is located in volume $\delta V_{\text {coll }}$ is expressed by

$$
p_{x_{1}, y_{1}, z_{1}}=\iint_{\delta V_{\text {coll }}} f(x, y, z) d x d y d z
$$

where $f(x, y, z)$ is the probability density function (pdf) of spatial node distribution in the volume $V$. For small values of $r$, the points $f(x, y, z)$ in $V$ can be approximated by $f\left(x_{1}, y_{1}, z_{1}\right)$. This gives the probability of a collision within the next infinitesimally small time interval, given that the first node's starting position is $\left(x_{1}, y_{1}, z_{1}\right)$, i.e.,

$$
p_{x_{1}, y_{1}, z_{1}} \approx \pi r_{12}^{2} v_{12} \delta t f\left(x_{1}, y_{1}, z_{1}\right)
$$

The first node's starting position can be changed. Therefore, by integrating $p_{x_{1}, y_{1}, z_{1}}$ over all starting positions of the first node, the probability of collision within the next infinitesimally small time interval $\delta t$ can be obtained as

$$
\begin{aligned}
p & =\iiint_{V} p_{x_{1}, y_{1}, z_{1}} f\left(x_{1}, y_{1}, z_{1}\right) d x_{1} d y_{1} d z_{1} \\
& \approx \pi r_{12}^{2} v_{12} \delta t \iiint_{V} f^{2}\left(x_{1}, y_{1}, z_{1}\right) d x_{1} d y_{1} d z_{1}
\end{aligned}
$$

(4) is specific to the relative speed $v_{12}$. Averaging the relative velocity over the velocity distributions of the nanonodes, the collision rate for nanodes can be approximated as

$$
R_{c} \approx \pi r_{12}^{2} E\left[v_{n n}\right] \iiint_{V} f^{2}\left(x_{1}, y_{1}, z_{1}\right) d x_{1} d y_{1} d z_{1}
$$

where $E\left[v_{n n}\right]$ is the average relative speed of the nanomachines. In order to evaluate the collision rate, the spatial node distribution $f(x, y, z)$, and average relative speed $E\left[v_{n n}\right]$ for the nanomachines moving according to Brownian motion are required. Note that the effect of average relative speed on performance is discussed in Section 6. In [16], it is shown that the steady-state node distribution in Brownian motion is uniform. Thus, $f(x, y, z)=1 / V$ and $R_{c}$ can be also approximated as

$$
\begin{aligned}
R_{c} & \approx \pi r_{12}^{2} E\left[v_{n n}\right] \iiint_{V} 1 / V^{2} d x_{1} d y_{1} d z_{1} \\
& \approx \frac{\pi r_{12}^{2} E\left[v_{n n}\right]}{V}
\end{aligned}
$$

Since the size of nanomachines are identical, $r_{12}$ can be given as $r_{12}=2 r$, where $r$ is the radius of a nanomachine. Then, from (6), the collision rate for nanomachines can be expressed as

$$
R_{c} \approx \frac{4 \pi r^{2} E\left[v_{n n}\right]}{V}
$$

The collision rate of an infostation and a nanomachine follows a similar argument except that infostations could have a different radius compared to nanomachines. For nanomachine-infostation $r_{12}$ is set as $r_{12}=r+r_{i}$, where $r_{i}$ is the radius of an infostation. Then, similar to the collision

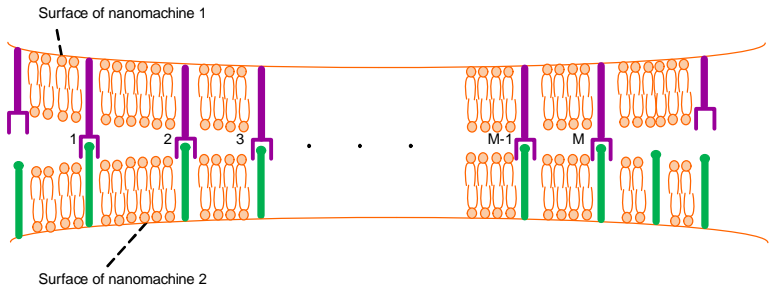

Fig. 3. Nanomachine Adhesion. Nanomachine 1 and Nanomachine 2 are attached to each other by $M$ ligand receptor pairs.

rate of nanomachines, the collision rate of an infostation and a nanomachine can be written as

$$
R_{i c} \approx \frac{\pi\left(r+r_{i}\right)^{2} E\left[v_{n i}\right]}{V}
$$

where $E\left[v_{n i}\right]$ is the average relative speed of the nanomachine and infostation.

The collision rates $R_{c}$ and $R_{i c}$ are essential to understand the interaction rate of nanomachines and infostations. However, the collision rates are not the ultimate parameters that affect the MAMNET performance since the collided nanomachines should also adhere with each other to enable molecular neuro-spike communication. Next, the adhesion of nanomachines is modeled.

\subsection{Adhesion of nanomachines}

After the collision between nanomachines, the collided nanomachines adhere with each other via the binding of the surface molecules called ligands and receptors, as shown in Fig. 3. In nature, the binding process of two cells, i.e., cell adhesion, is an important issue. Cell adhesion is involved in a variety of processes such as migration, invasion, embryogenesis, wound healing and cell-to-cell communication [19].

In this paper, the natural cellular adhesion paradigm is adopted to enable the adhesion of nanomachines. In literature, there exists several research efforts on modeling the adhesion between cells [9], [17]. Here, the adhesion model developed in [9] is adopted. Accordingly, nanomachines are assumed to have ligands and receptors which mediate adhesion. The adhesion process is heavily affected by the density of ligands and receptors and the contact area of the collided nanomachines.

Adhesion is considered as a random event and the state of the system is considered as a probability vector $\left[p_{0}, p_{1}, \ldots, p_{n}, \ldots, p_{A_{c} m_{\min }}\right]$ where $m_{\min }$ is the minimum of surface densities of receptors and ligands and $A_{c}$ is the area of contact [9]. In other words, adhesion could be mediated by any number of bonds ranging from 0 to $A_{c} m_{\min }$. For forming any number of bonds, there is a defined likelihood given by $p_{n}$. Here, nanomachines are assumed to adhere when at least $c$ bonds are formed between them. Hence, our aim is to derive the probability that nanomachines adhere via at least $c$ bonds, and the closed-form expression of $p_{n}$ is needed in deriving this. In [9], a closed-form expression 
is found for $p_{n}$ under a set of certain assumptions for two cells, such as either ligands or receptors outnumbers the other one. When two nanomachines are just brought together $(t=0)$, there is no bond, hence,

$$
p_{n}(0)= \begin{cases}1 & , n=0 \\ 0 & , n \neq 0\end{cases}
$$

With the contact of nanomachines, bonds start to form according to a single step reversible reaction. The chemical reaction of $v_{r}$ receptors (designated $M_{r}$ ) binding to $v_{l}$ ligands (designated $M_{l}$ ) to form $v_{b}$ bonds (designated $M_{b}$ ) can be expressed as

$$
v_{r} M_{r}+v_{l} M_{l} \stackrel{k_{f}^{0}, k_{r}^{0}}{\longrightarrow} v_{b} M_{b}
$$

where $k_{f}^{0}$ and $k_{r}^{0}$ are the forward and reverse rate constants, respectively.

In the stochastic model of ligand-receptor binding process, the state of the system, described by the single step reversible chemical reaction in (10), is represented by the probability vector $\left[p_{0}, p_{1}, \ldots, p_{n}, \ldots, p_{A_{c} m_{m i n}}\right]$. In [15], a stochastic model of ligand-receptor binding process is discussed and master equations are derived in order to describe the state of the system. Actually, these master equations can be used to describe the rate of change in probability $p_{n}(t)$. However, it may not possible to obtain closed-form solutions for the master equations. However, in the current literature, two simplified versions of master equations are discussed in order to obtain closed-form expressions. In [9], a simplified version is discussed under the condition that either ligands or receptors excessively outnumber the other one. Under that condition and with $v_{r}=v_{l}=v_{b}=1$, i.e., a bond is formed by the binding of a ligand to a receptor, the master equations can be simplified as [9]

$$
\begin{array}{r}
\frac{d p_{n}}{d t}=\left[A_{c} m_{\min }-(n-1)\right] m_{\max } k_{f}^{0} p_{n-1}- \\
-\left[\left(A_{c} m_{\min }-n\right) m_{\max } k_{f}^{0}+n k_{r}^{0}\right] p_{n}+(n+1) k_{r}^{0} p_{n+1}
\end{array}
$$

Assuming either ligands or receptors excessively outnumber the other one, $p_{n}(t)$ is found to be in the form of binomial distribution and can be given as [9]

$$
p_{n}(t)=\left(\begin{array}{c}
A_{c} m_{\min } \\
n
\end{array}\right)[p(t)]^{n}[1-p(t)]^{A_{c} m_{\min }-n}
$$

where $p(t)$ is the probability of forming one bond given by

$$
p(t)=\frac{1-e^{-k t}}{1+\left(m_{\max } K_{a}^{0}\right)^{-1}}
$$

where $K_{a}^{0}=k_{f}^{0} / k_{r}^{0}$ is the equilibrium association constant and $k=m_{\max } k_{f}^{0}+k_{r}^{0}$ is the overall rate of reaction.

After the collision event between two nanomachines, they are assumed to stay in contact with each other during an average contact duration $\tau_{c}$. $\tau_{c}$ is affected by the physical properties of the environment, relative velocities and physical surface properties of the nanomachines. The effect of $\tau_{c}$ on performance is discussed in Section 6. We also

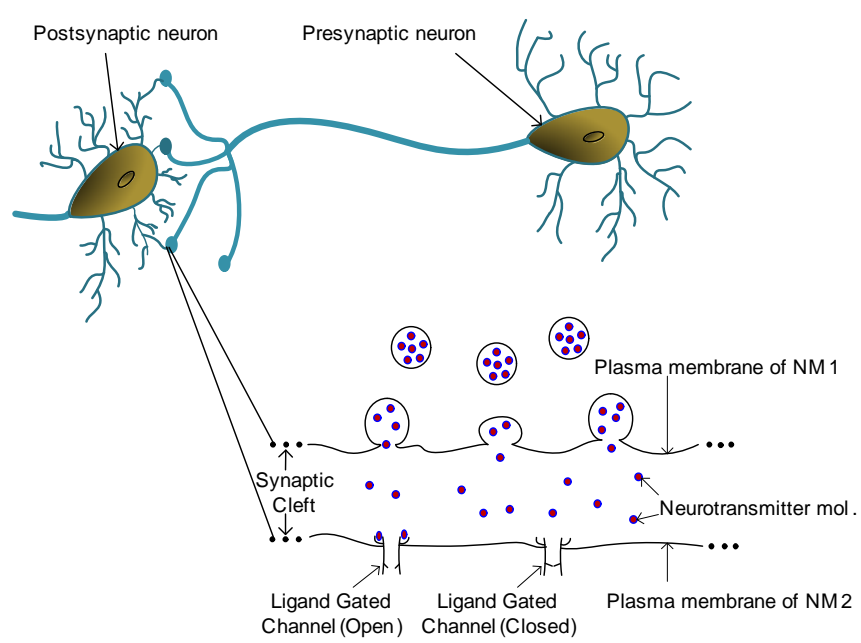

Fig. 4. Neuro-spike transmission between nanomachines. Neurotransmitters emitted from the transmitter nanomachine (TN), diffuse through the gap between the nanomachines and reach the receiver nanomachine (RN).

assume that the collided nanomachines adhere with each other if at least $c$ bonds are formed during a contact duration $\tau_{c}$. Hence, the probability that the collided nanomachines adhere with each other, i.e., $R_{a}$, can be given as

$$
R_{a}=1-\sum_{i=0}^{c-1} p_{i}\left(\tau_{c}\right)
$$

Note that we do not present a different adhesion rate for the nanomachine-infostation interaction. This is because the physical properties used in the derivation of adhesion rates are the same for both nanomachines and infostations. The adhesion probability derived in this section is very important to understand the interaction of nanomachines. The communication between nanomachines can only be possible after successful adhesion.

\section{Molecular Neuro-Spike Communi- CATION}

In this section, molecular neuro-spike communication is explained in detail. First, the basics of molecular neurospike communication are introduced. Then, its channel capacity and error probability are analytically investigated. Finally, successful information transmission probability in the molecular neuro-spike communication channel is derived to be used for the performance evaluation of MAMNET.

\subsection{Basics of Molecular Neuro-Spike Communica- tion}

When a nanomachine that has an information collide and successfully adhere to another nanomachine, it transmits its information to this nanomachine by means of molecular neuro-spike communication. The nanomachine that transmits the information is called the transmitter nanomachine 


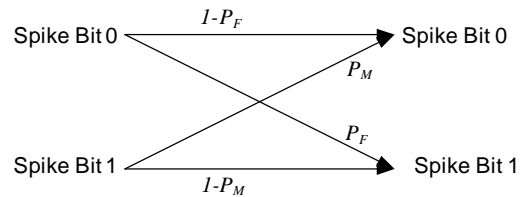

Fig. 5. Channel model of synaptic transmission. Transmission probabilities are shown for the binary channel.

(TN) and the nanomachine that receives the information is called the receiver nanomachine (RN). After the adhesion of nanomachines, there exists a small gap, synapse, between the TN and RN. This synapse is the communication media for nanomachines. Since TN and RN cannot remain adhered for a long time, a fast and reliable communication paradigm is needed to enable the information transmission between nanomachines. Among the existing inter-cellular communication paradigms, the communication between neuron cells is the fastest one. Therefore, in our model, we adopt the principles of neural communication to enable the information transmission among nanomachines.

In human body, there already exist at least two types of natural synapses between cells, namely, the neuronal synapse and the immunological synapse. These specialized contacts directly transfer highly controlled secretory signals between the adjacent cells [18]. The neuronal synapse is formed between the neurons and it retains the connectivity of the neuron cells throughout the life, whereas the immunological synapse is formed by the instant contacts of immune cells. Although nervous system and immune system have totally different roles in human life, in both systems cells need to communicate, and this is accomplished in the synapse. Therefore, synapses play a critical role in the cell-to-cell communication.

In nervous system, neural signals propagate in the form of electrochemical waves. These electrochemical waves are basically action potentials that propagate along axons and transmitted to other neurons. Action potential is basically an electrical pulse which has approximately 80 $\mathrm{mV}$ amplitude. At synapse, action potentials or spikes are electrochemically transmitted from one neuron to another. The signal is transmitted by means of chemical messengers called neurotransmitters. Neurotransmitters are contained in vesicles. The coming pulse or action potential, releases the neurotransmitters on the pre-synaptic neuron, and then, the neurotransmitters bind to the receptors on the post-synaptic neuron. Binding of a neurotransmitter to a receptor opens a channel which lies between inside and outside of the postsynaptic neuron. These channels allow the flow of ions to the neuron. The net movement of ions causes the membrane potential of the post-synaptic neuron to change rapidly. In this way, spikes or action potentials are transmitted to the next neuron.

In this work, we adopt the principles of neural communication to develop molecular neuro-spike communication model. The communication between nanomachines is realized with spike transmission as shown in Fig. 4. The communication process based on molecular neuro-spike communication involves the following steps:

1) Encoding: Similar to the traditional digital communication, we define 2 bit levels, i.e., spike bit 0 and spike bit 1 corresponding to logic 0 and 1 . The information is encoded on the concentration of the released neurotransmitter molecules. For spike bit 1, neurotransmitters are released to the channel, whereas for spike bit 0 no releasing occurs.

2) Transmission: Actually, this process initiates the electrochemical signaling. Whenever a TN wants to send a spike bit 1 , it simply activates the release of vesicles that contain neurotransmitters.

3) Signal Propagation: The released neurotransmitters propagate in the synaptic channel formed between the adherent nanomachines. The aim of the neurotransmitters is to bind to the receptors on the RN.

4) Reception: The neurotransmitters released into the synaptic channel reach the RN and bind to the receptors on the RN membrane as in Fig. 4. The binding of neurotransmitters to receptors opens ligand gated channels that let the flow of ions into or out of the $\mathrm{RN}$. The flow of ions changes the membrane voltage.

5) Decoding: RN nanomachine monitors the plasma membrane voltage for certain time periods. If $\mathrm{RN}$ observes a rapid change in the membrane voltage it decides the received bit as spike bit 1 , otherwise the decision is spike bit 0 .

In an ideal channel, the spike bit sent by a TN should be perfectly received by the receiver nanomachine. However, in practice there exist two kinds of errors as shown in Fig. 5 , namely, false alarm and miss detection errors. The false alarm and miss detection probabilities, i.e., $P_{F}$ and $P_{M}$, respectively, can be defined as

$$
\begin{aligned}
& P_{F}=\operatorname{Prob}[Y=1 \mid X=0] \\
& P_{M}=\operatorname{Prob}[Y=0 \mid X=1]
\end{aligned}
$$

where $X$ and $Y$ are the input and output symbols, respectively. In order to derive these probabilities, a close look at neuronal synapse would be helpful. In [20], a cortical synapse is modeled as a binary channel and the information-theoretical capacity is derived for two different coding paradigms, i.e., signal estimation and signal detection. Although this synaptic transmission model ignores certain aspects like paired-pulse facilitation, vesicle depletion, calcium buffering, it represents a simplified and compact picture of synaptic transmission. Therefore, we adopt the synaptic transmission model in [20] and accordingly derive the channel capacity expression.

In Fig. 6, the block diagram of the channel model of synaptic transmission is shown. The input to the channel, $X(t)$, is a spike bit. If the input is spike bit 1 , vesicles containing neurotransmitters are released by the $\mathrm{TN}$ to the synaptic cleft. For spike bit 0 , no vesicles are released.

Neurotransmitters binding to the receptors on the RN membrane open channels that let the flow of ions. The flow of ions create an Excitatory Postsynaptic Potential 


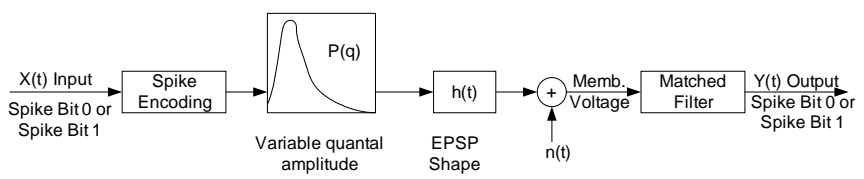

Fig. 6. Block diagram of the channel model of synaptic transmission [20]. Spikes sent from the transmitter nanomachines pass through a filtering process and an additive gaussian noise is corrupted at the post-synaptic membrane. The decision by the receiver nanomachine is based on the matched filter.

(EPSP) on the RN. In Fig. 6, EPSP profile or shape of $\mathrm{RN}$ is modeled by a filter with impulse response $h(t)$ [20]. In Fig. 6, the random variable $q$, which has probability density $P(q)$, models the variability in the size of EPSP. The number of neurotransmitter molecules released by the $\mathrm{TN}$, the number of available postsynaptic receptors on the $\mathrm{RN}$ and several other factors affect this variability. In Fig. 6 , there exists also an additive noise, $n(t)$, coupled to the response of RN. The noise $n(t)$ accounts for the other noise sources in the membrane of the RN such as thermal noise and channel noise [21].

\subsection{Information Theoretical Capacity of Molecular Neuro-Spike Communication}

In molecular neuro-spike communication, at the receiver side, the aim of $\mathrm{RN}$ is to optimally detect the presence or absence of a single spike through the knowledge of membrane voltage $V_{m}(t)$. In [20], it is shown that the optimal decision rule for spike detection is to compare the correlation $r$ between $V_{m}(t)$ and $h(t)$ to a threshold $\theta$. Thus, the decision rule can be written as

$$
\begin{aligned}
& r \geq \theta \Rightarrow Y=1, \\
& r<\theta \Rightarrow Y=0
\end{aligned}
$$

With this decision rule, the false alarm and miss detection probabilities are written as

$$
\begin{aligned}
P_{F} & =\operatorname{Prob}[r \geq \theta \mid X=0] \\
P_{M} & =\operatorname{Prob}[r<\theta \mid X=1]
\end{aligned}
$$

In [20], the probabilities of miss detection and false alarm are derived for both stochastic and deterministic vesicle release processes. In our model, we use the probabilities derived for deterministic vesicle release, i.e.,

$$
\begin{gathered}
P_{F}=\frac{1}{2}[1-\operatorname{Erf}(\theta)] \\
P_{M}=\frac{1}{2}\left[1+\int_{0}^{\infty} \operatorname{Erf}(\theta-q \sqrt{S N R}) P(q) d q\right]
\end{gathered}
$$

where $\operatorname{Erf}(x)$ is the error function and $S N R$ is the signalto-noise ratio on the post-synaptic potential.

Based on the false alarm and miss detection probabilities, the molecular neuro-spike channel can now be modeled as a binary symmetric channel. TN emits spike bit 1 with probability $p_{1}$ and spike bit 0 with probability $\left(1-p_{1}\right)$ Then, the probability of error for one spike bit can be written as

$$
P_{E}=p_{1} P_{M}+\left(1-p_{1}\right) P_{F}
$$

The value of $P_{E}$ depends both on the threshold $\theta$ and $S N R$. For small values of $\theta, P_{M}$ is low whereas $P_{F}$ is high. On the other hand, for large values of $\theta, P_{F}$ is low whereas $P_{M}$ is high. Therefore, $P_{E}$ should be evaluated with varying $\theta$ in order to find an optimum value for $\theta$. In Fig. 7(a), the dependencies of $P_{E}, P_{M}$ and $P_{F}$ on $\theta$ are shown for a fixed value of $S N R=10$ assuming an equallylikely prior probability $\left(p_{1}=0.5\right)$. With decreasing $S N R$, the probability of error increases. Furthermore, $S N R$ also has an impact on the optimum value of threshold. As can be seen in Fig. 7(b), the optimum value of $\theta$ becomes closer to 0 as the value of $S N R$ decreases.

The performance of the molecular neuro-spike communication can be further quantified by the mutual information. The transition matrix of the molecular neuro-spike channel can be written as follows

$$
P(Y \mid X)=\left(\begin{array}{cc}
p_{1}\left(1-P_{M}\right) & \left(1-p_{1}\right) P_{F} \\
p_{1} P_{M} & \left(1-p_{1}\right)\left(1-P_{F}\right)
\end{array}\right)
$$

Using the transition matrix in (21), the mutual information $I(X ; Y)$ between $\mathrm{X}$ and $\mathrm{Y}$ can be derived as follows

$$
\begin{aligned}
I(X ; Y) & =H(Y)-H(Y \mid X) \\
& =H\left(p_{1} P_{M}+\left(1-p_{1}\right)\left(1-P_{F}\right)\right) \\
& -\left(p_{1} H\left(P_{M}\right)+\left(1-p_{1}\right) H\left(P_{F}\right)\right)
\end{aligned}
$$

where $H(z)$ denotes the binary entropy function $H(z)=$ $-z \log _{2}(z)-(1-z) \log _{2}(1-z)$. Mutual information corresponds to how much one can guess about the input with the knowledge of the output. $\theta$ and $S N R$ have a direct effect on the value of mutual information. For low $S N R$ values, mutual information can be very low. However, as $S N R$ increases the mutual information increases as well, as shown in Fig. 7(c). The optimum value of $\theta$, changes with $S N R$.

In a synaptic channel, spikes can be transmitted by $3 \mathrm{~ms}$ differences which is composed of a $0.5 \mathrm{~ms}$ action potential pulse and $2.5 \mathrm{~ms}$ of recovery time. Thus, a theoretical bandwidth of 333 bps can be reached. This corresponds to an ideal synaptic channel with $I(X ; Y)=1$, i.e., every bit transmitted contains exactly 1 bit of information. However, in practice there exist channel errors and this rate cannot be achieved.

The capacity of the molecular-neuro spike channel can be obtained by maximizing the mutual information over all input distributions, i.e.,

$$
C=\max _{p_{X}}(I(X ; Y))
$$

As can be seen in Fig. 7(d), the mutual information can be at most 0.57 for the channel under consideration. This corresponds to a bandwidth of approximately $333 \times 0.57 \approx$ 190 bps. Even if it is slightly larger than half of the ideal bandwidth of an ideal synaptic channel, it is a considerably high bandwidth. 


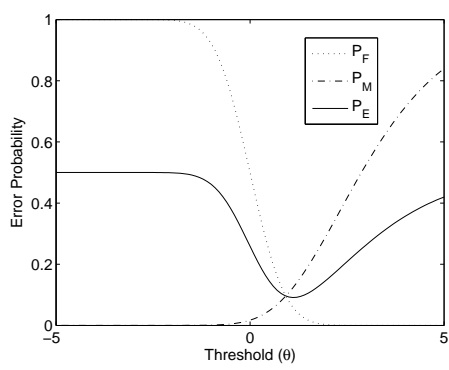

(a)

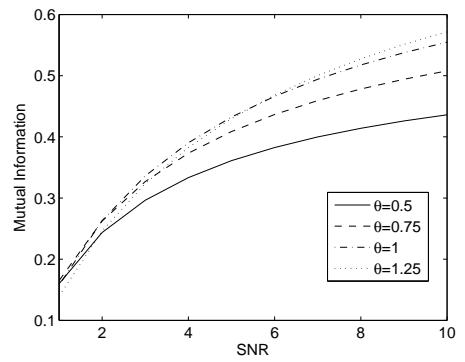

(c)

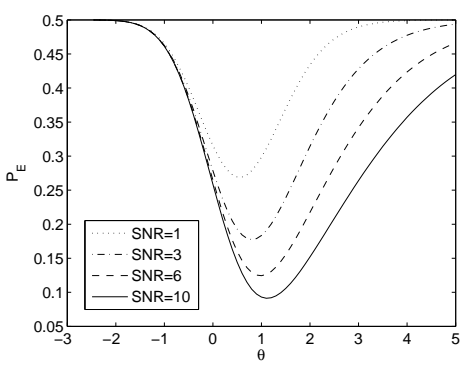

(b)

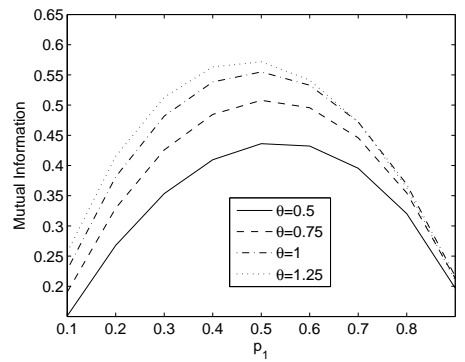

(d)

Fig. 7. (a) False alarm, miss and channel error probabilities in molecular neuro-spike channel for a fixed value of $S N R=10$ with varying $\theta,\left(p_{1}=0.5\right)$. (b) Channel error probability in molecular neuro-spike channel with varying $\theta$ for different values of $S N R,\left(p_{1}=0.5\right)$. (c) Mutual information with varying $S N R$ for different values of $\theta,\left(p_{1}=0.5\right)$. (d) Mutual information with varying $p_{1}$ for different values of $\theta,(S N R=10)$.

By analyzing single spike bit error probability and channel capacity expressions, it is shown that molecular neuro-spike channel promises a reliable and fast communication. Therefore, molecular neuro-spike communication stands as a promising solution for the communication of adherent nanomachines in MAMNET. In evaluating the performance of MAMNET, information transmission probability is needed. Information transmission probability can be described as the successful transmission of all the information from $\mathrm{TN}$ to $\mathrm{RN}$.

The information collected by the $\mathrm{TN}$ is encoded to spikes and sent to the RN. The probability of successful information transmission is analogous to the successful transmission of all spike bits in the message. Hence, successful information transmission probability is

$$
R_{t}=\left(1-P_{E}\right)^{n}
$$

where $n$ is the number of spike bits contained in the message. The probability in (24) is used in the analytical model of MAMNET.

\section{Mathematical Model of Mobile Ad Hoc MOLECULAR NANONETWORK}

In this section, a closed form expression for average message delivery delay is also derived for MAMNET. Furthermore, average throughput and system throughput expressions are obtained. The propagation of a single message is modeled using the principles of epidemic disease spreading. For this purpose, a store-carry-forward scheme is proposed. When a nanomachine having an information

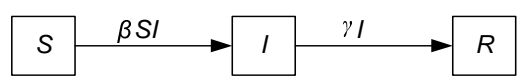

Fig. 8. S-I-R Model. Markov chain model of an epidemic disease spreading. S, I, R represents the susceptible, infected and the recovered states respectively.

encounters with another nanomachine that does not have a copy of the information, it forwards the information to this nanomachine.

In mobile ad hoc networks with intermittent connectivity, many routing protocols inspired by epidemic disease spreading have been previously developed [22], [23], [24]. Similar to these works, inspiring by epidemic disease spreading nanomachines can be in three different states, i.e., infected, suspicious and recovered. The message which should be transmitted to an infostation, is analogous to the agent of a disease. An infected nanomachine is the one that has a copy of the message. A nanomachine is said to be suspicious when it does not have a copy of the message, but could potentially acquire a copy of the message from the infected ones. A nanomachine is recovered after it has offloaded the message to the infostation.

Our model is developed upon the Markov model devised for the basic epidemic disease spreading [25]. In Fig. 8, $S, I$ and $R$ are the susceptible, infected and recovered states, respectively. $S(t), I(t)$ and $R(t)$ are the numbers of nanomachines in the susceptible, infected and recovered states, respectively. In MAMNET, $\beta$ represents the rate of contacts, that ends with successful transmission between two nanomachines, per nanomachine and $\gamma$ represents the 
rate of contacts, that ends with successful transmission, between a nanomachine and an infostation, per nanomachine. The model is based on three assumptions, which can be adopted for our model as follows.

i. An infected nanomachine makes contacts, that are ended with the successful transmission of message, with $\beta(N-1)$ others per unit time where $N$ represents the total number of nanomachines in MAMNET. $\beta$ expresses the total rate including collision $\left(R_{c}\right)$, adhesion $\left(R_{a}\right)$ and transmission rates $\left(R_{t}\right)$ for nanomachines. Therefore, $\beta=$ $R_{c} \times R_{a} \times R_{t}$. Since $S /(N-1)$ proportion of these contacts are with the suspicious ones, the number of infections per unit time can be derived as $(\beta(N-1))(S /(N-1)) I=\beta S I$.

ii. $\quad \gamma$ expresses the rate with which infected nanomachines become non-infected (recovered) and therefore, it contains infostation-nanomachine collision, adhesion and transmission rates. Therefore, $\gamma$ can be given as $\gamma=R_{i c} \times R_{a} \times R_{t}$. If there are more than one infostation, the parameter $\gamma$ should be multiplied by the number of infostations. This is because, the delivery of message to any infostation means recovery.

iii. The total number of individuals in the system is constant.

The message delivery delay $T_{d}$ is defined as the time elapsed from an information message is first generated by a nanomachine to the time when this message is first offloaded to an infostation. The initial conditions for the system can be defined as follows. At time $t=0$, only one nanomachine is infected, i.e., $I(0)=1, S(0)=N-1$. Until offloading, the entire number of nanomachines $N$ will be contained in either $I$ state or $S$ state, i.e., $S+I=N, R(t)=$ 0 for $t<T_{d}$. At the time of offloading, $R\left(T_{d}\right)=1$.

In order to find message delay distribution, it should be required that the transient solution of the Markov Chain given in Fig. 8 and modeled as

$$
\frac{d I}{d t}=\beta S I=\beta N I-\beta I^{2}
$$

For $I(0)=1$, the solution of (25) is given in [22] as

$$
I(t)=\frac{N}{1+(N-1) e^{-\beta N t}}
$$

Using (26), the cumulative distribution function (CDF) of the message delay can be found. The CDF describes the probability that the message is delivered to an infostation by time $t$ and denoted by $F(t)=\operatorname{Pr}\left(T_{d}<t\right)$. The differential equation for $F(t)$ can be written as [22]

$$
\frac{d F}{d t}=\lim _{\epsilon \rightarrow 0} \frac{F(t+\epsilon)-F(t)}{\epsilon}
$$

where $\epsilon$ is an arbitrary positive small number close to 0 . Note that, $F(t)=1-\operatorname{Pr}\left(T_{d}>t\right)$ and $F(t+\epsilon)=$ $1-\operatorname{Pr}\left(T_{d}>t+\epsilon\right)$. In order to find $F(t)$ using (27), the probability $\operatorname{Pr}(T \geq t+\epsilon)$ is needed. Assuming
$\operatorname{Pr}($ event in $[0, t))$ is independent of $\operatorname{Pr}($ event in $[t, t+\epsilon))$,

$$
\begin{aligned}
& \operatorname{Pr}\left(T_{d}>t+\epsilon \mid T_{d}>t\right) \\
& =1-\operatorname{Pr}\left(t<T_{d}<t+\epsilon \mid T_{d}>t\right) \\
& =1-\epsilon \gamma I(t)
\end{aligned}
$$

From (28), $\operatorname{Pr}\left(T_{d}>t+\epsilon\right)$ can be calculated as

$$
\begin{aligned}
\operatorname{Pr}\left(T_{d}>t+\epsilon\right) & =\operatorname{Pr}\left(T_{d}>t\right) \operatorname{Pr}\left(T_{d}>t+\epsilon \mid T_{d}>t\right) \\
& =\operatorname{Pr}\left(T_{d}>t\right)(1-\epsilon \gamma I(t))
\end{aligned}
$$

This can be used to derive the differential equation for $F(t)$, i.e.,

$$
\begin{aligned}
\frac{d F}{d t} & =\lim _{\epsilon \rightarrow 0} \frac{\left[1-\operatorname{Pr}\left(T_{d}>t+\epsilon\right)\right]-\left[1-\operatorname{Pr}\left(T_{d}>t\right)\right]}{\epsilon} \\
& =\lim _{\epsilon \rightarrow 0} \frac{-1}{\epsilon} \operatorname{Pr}\left(T_{d}>t\right)(1-\epsilon \gamma I(t)-1) \\
& =\gamma I(t) \operatorname{Pr}\left(T_{d}>t\right) \\
& =\gamma \frac{N}{1+e^{-\beta N t}(N-1)}[1-F(t)]
\end{aligned}
$$

For the solution of (30), an initial condition is required. Note that, $F(0)$ represents the probability that the nanomachine which generates the message is adherent with infostation such that it can directly transmit the message to the infostation. Therefore, $F(0)$ can be given by

$$
F(0)=\frac{4 \pi\left(r_{i}^{3}-r^{3}\right)}{3 V}
$$

Using $F(0), F(t)$ can be derived as

$$
F(t)=1-K\left(\frac{N-1}{N-1+e^{\beta N t}}\right)^{\frac{\gamma}{\beta}}
$$

where $K=\left[\frac{N-1}{N}\right]^{\frac{-\gamma}{\beta}}[1-F(0)]$. Hence, the probability that a message is delivered to an infostation for a given time can be obtained. Conversely, the average time needed to deliver the information to an infostation can also be calculated. Average delivery delay can be found using CDF of message delivery delay, i.e.,

$E\left[T_{d}\right]=\int_{0}^{\infty} 1-F(t) d t=\int_{0}^{\infty} K\left(\frac{N-1}{N-1+e^{\beta N t}}\right)^{\frac{\gamma}{\beta}} d t$

Assuming $\gamma / \beta \in N^{+}, E\left[T_{d}\right]$ can be analytically expressed as

$$
E\left[T_{d}\right]= \begin{cases}\frac{F(0) \ln N}{\gamma(N-1)} & , \gamma / \beta=1 \\ \frac{F(0)(1-N)}{\beta N N^{2}}\left[\sum_{k=2}^{\gamma / \beta} \frac{\beta}{\beta+\gamma-k \beta}\left(\frac{N}{N-1}\right)^{k}-\right. & \\ \left.\left(\frac{N}{N-1}\right)^{1+\gamma / \beta} \log N\right] & , \gamma / \beta \geq 2\end{cases}
$$

Note that, the analytical result in (33) are only valid for $\gamma / \beta \in N^{+}$. On the other hand, by numerically evaluating the integral in (33), $E\left[T_{d}\right]$ can be calculated for any $\gamma$ and $\beta$. The numerical results for average delivery delay are presented in Section 6.

Using the average delivery delay, average throughput of MAMNET can also be calculated. Considering from an infostation point of view, a message encoded by $n$ spike bits 
TABLE 1 Simulation Parameters

\begin{tabular}{l|l}
\hline \hline Radius of a nanomachine $(r)$ & $7.5(\mu \mathrm{m})$ \\
\hline Radius of an infostation $\left(r_{i}\right)$ & $15(\mu \mathrm{m})$ \\
\hline $\begin{array}{l}\text { Average relative speed of nanoma- } \\
\text { chines }\left(E\left[v_{n n}\right]\right)\end{array}$ & $1(\mu \mathrm{m} / \mathrm{sec})$ \\
\hline $\begin{array}{l}\text { Average relative speed of nanoma- } \\
\text { chines and infostation }\left(E\left[v_{n i}\right]\right)\end{array}$ & $1(\mu \mathrm{m} / \mathrm{sec})$ \\
\hline Volume $(V)$ & $1000000\left(\mu \mathrm{m}^{3}\right)$ \\
\hline Area of contact $\left(A_{c}\right)$ & $3\left(\mu \mathrm{m}^{2}\right)$ \\
\hline Surface density of receptors $\left(m_{r}\right)$ & $100\left(1 / \mu \mathrm{m}^{2}\right)$ \\
\hline Surface density of ligands $\left(m_{l}\right)$ & $75\left(1 / \mu \mathrm{m}^{2}\right)$ \\
\hline Forward binding rate $\left(k_{f}^{0}\right)$ & $\begin{array}{l}1.32 \times \\
\left(\mu \mathrm{m}^{2} / \mathrm{sec}\right)\end{array}$ \\
\hline Reverse binding rate $\left(k_{r}^{0}\right)$ & $0.36(1 / \mathrm{sec})$ \\
\hline Contact time $\tau_{c}$ & $1(\mathrm{sec})$ \\
\hline $\begin{array}{l}\text { Minimum number of required bonds for } \\
\text { proper adhesion }(c)\end{array}$ & 40 \\
\hline $\begin{array}{l}\text { Prior probability of sending spike bit } 1 \\
\left(p_{1}\right)\end{array}$ & 0.5 \\
\hline Threshold for decision of spike bit $(\theta)$ & 1.1 \\
\hline Signal to noise ration on RN $(S N R)$ & 10 \\
\hline Length of message $(n)$ & $8(\mathrm{bits})$ \\
\hline Number of nanomachines $(N)$ & 20 \\
\hline
\end{tabular}

is transmitted from a nanomachine source to an infostation in an average time of $E\left[T_{d}\right]$. Hence, the average throughput of MAMNET can be described as

$$
T_{\text {avg }}=\frac{n}{E\left[T_{d}\right]}
$$

On the other hand, in the whole system, an average number of $I\left(E\left[T_{d}\right]\right)$ nanomachines get infected, i.e., apart from source, an average number of $I\left(E\left[T_{d}\right]\right)-1$ copies of the message exist in the network. This means that, an average of $\left(I\left(E\left[T_{d}\right]\right)-1\right) \times n$ spike bits are transmitted between nanomachines until the message arrives into an infostation. Also accounting for the $n$ spike bits transmitted to infostation, the system throughput can be written as

$$
T_{\text {sys }}=\frac{I\left(E\left[T_{d}\right]\right) n}{E\left[T_{d}\right]}
$$

Consequently, all aspects of MAMNET are covered by this model. The expressions derived for $E\left[T_{d}\right], T_{a v g}$ and $T_{s y s}$ clearly describe the performance of MAMNET. These expressions can be used to investigate the feasibility and evaluate performance of MAMNET. Using the model derived above, next, the performance of MAMNET is evaluated.

\section{Performance Analysis of Mobile Ad Hoc Molecular Nanonetwork}

In this section, we evaluate the performance of MAMNET by using average message delay, average throughput and system throughput expressions derived in previous section. We use MATLAB to obtain analytical results. The aim is to investigate delay and throughput performance and gain insight on the feasibility of MAMNET. The parameters used in the analysis are given in Table 1. In the following sections, the effect of these parameters is investigated.

\subsection{Effect of Relative Speed and Size of Nanoma- chines}

We first observe the effect of relative speed and size of nanomachines on average delay, average throughput and system throughput. Nanomachines and infostation are contained in a volume of $10^{6} \mu \mathrm{m}^{3}$. Nanomachine size is on the order of typical mammalian cells, i.e. having a diameter of 5-10 $\mu \mathrm{m}$. Since infostations have more complex roles than nanomachines, they are assumed to have larger dimensions than nanomachines, e.g., around 10-20 $\mu \mathrm{m}$.

In Fig. 9(a), Fig. 9(b) and Fig. 9(c), average message delay, average throughput and system throughput are shown for different $r$ with varying $E\left[v_{n n}\right]$, respectively. For $r=7.5 \mu \mathrm{m}$, average message delivery delay ranges from a few hundreds to a thousand seconds. Although, these can be considered as huge message delays for traditional networks, these values are very reasonable for MAMNET. The volume in nanomachines contained is 565 times larger than a nanomachine volume. Furthermore, the average relative speed of nanomachines is very low. Hence, the collision rate in MAMNET is low, e.g., with the values specified in Table 1, on average, only 14 collisions occur between nanomachines in 1000 seconds. Therefore, it is reasonable to end up with message delays on the order of a few thousand seconds. The system throughput follows a similar argument. Considering the effect of low collision rate and the information message is encoded by 8 spike bits, it is also reasonable to encounter with a system throughput on the order of few spike bits per 10 seconds.

In Fig. 9(a), average message delivery delay decreases with increasing relative speed of nanomachines. On the other hand, in Fig. 9(b) and Fig. 9(c), average throughput and system throughput increase with $E\left[v_{n n}\right]$. This is because, by increasing the relative speed of nanomachines the collision rate is amplified and this causes more nanomachines to get infected. Therefore, the number of information transfers in MAMNET increases, which yields a higher throughput. Moreover, the message is delivered to an infostation more quickly.

Fig. 9(a), Fig. 9(b) and Fig. 9(c) also show the effect of $r$ on $E\left[T_{d}\right], T_{a v g}$ and $T_{s y s}$, respectively. The increase in $r$ also causes collision rate to increase and this yields a higher throughput and lower message delivery delay. Actually, $r$ is analogous to the transmission range of mobile nodes in traditional mobile ad hoc networks. The larger the transmission range, the higher the probability of meeting of nodes. However, the transmission range is limited in traditional mobile ad hoc networks because of limited power. Similarly, it seems reasonable to make larger nanomachines to increase the performance, however, one has to consider that these nanomachines can be used in applications where the dimensions of the nanomachines is a critical issue. 


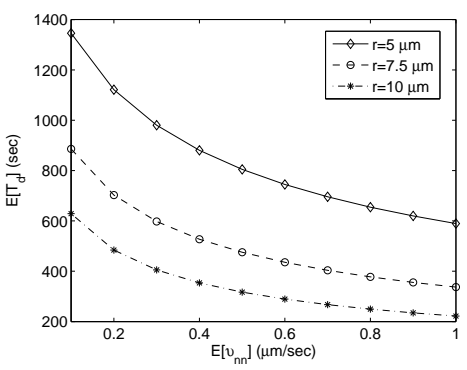

(a)

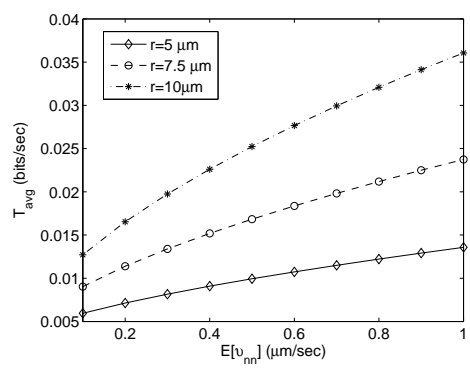

(b)

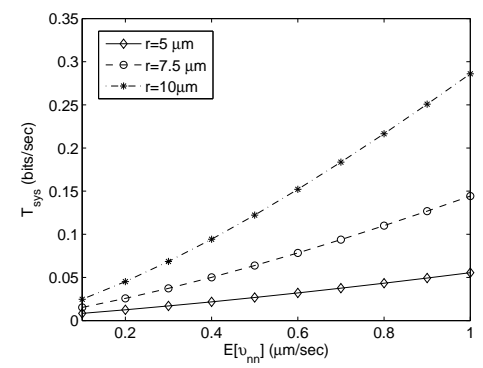

(c)

Fig. 9. (a) Average message delay (b) average throughput (c) system throughput with varying average relative speed of nanomachines for different values of nanomachine radius.

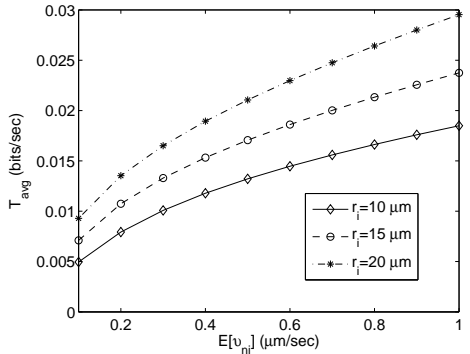

(a)

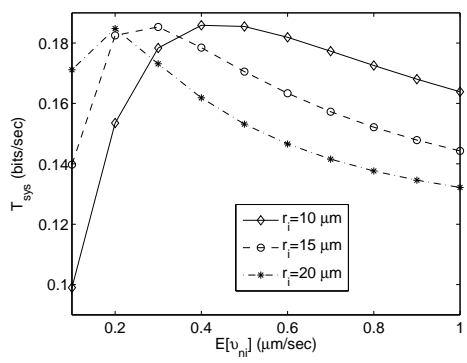

(c)

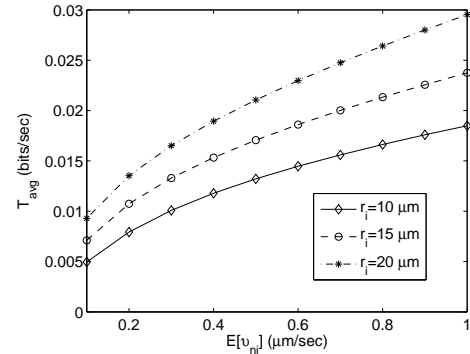

(b)

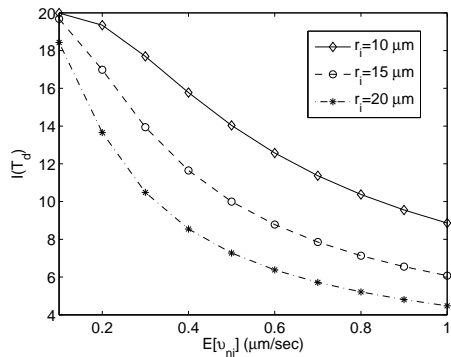

(d)

Fig. 10. For varying average relative speed of nanomachine and infostation for different values of infostation radius, the plot of (a) Average message delay. (b) Average throughput. (c) System throughput. (d) Average number of infected nanomachines at the instant of message delivery.

\subsection{Effect of Relative Speed and Size of Infosta- tions}

Here, the effects of relative speed and the size of infostations on performance are discussed. $E\left[T_{d}\right], T_{a v g}$ and $T_{\text {sys }}$ are shown for different infostation radius values with varying relative speed in Fig. 10(a), Fig. 10(b) and Fig. 10 (c), respectively. Increasing $r_{i}$ and $E\left[v_{n i}\right]$ increases the collision rate $R_{i c}$ of nanomachines and infostation. Therefore, the interactions between nanomachines and infostation increase which further yield a better delay performance and higher average throughput.

However, the system throughput performance does not have such a direct relation. This is because, system throughput is defined as the average number of spike bits transmitted in the system until the offloading of a message. For $E\left[v_{n i}\right]=0.1$, the collision rate of nanomachines and infostations are extremely low, creating a high message delivery delay. Until the delivery of the message almost all of the nanomachines get infected. Doubling $E\left[v_{n i}\right]$ causes the message delivery delay to decrease significantly. However, the number of infected nanomachines does not change at the same pace (Fig. 10(d)). Thus, the throughput increases significantly because of the significant decrease in average message delivery delay. Further increasing of $E\left[v_{n i}\right]$ causes message delivery delay to decrease, and also the number of infected nanomachines decreases as well. Therefore, the system throughput performance decreases.

\subsection{Effect of Contact Time}

In Fig. 11(a), average message delay and system throughput are shown with varying contact time. A long contact time yields a high adhesion probability and more of the collisions result with successful adhesion. Therefore, $E\left[T_{d}\right]$ decreases and $T_{\text {sys }}$ increases with increasing $\tau_{c}$. In Fig. 11(a), after a certain value of $\tau_{c}$, the performance of MAMNET does not change. The reason can be observed more clearly 
in Fig. 11(b). The contact time of nanonodes has only an effect on the adhesion probability. Actually, at $\tau_{c}=1.1$ second, the adhesion probability becomes 1 , and after that point, the adhesion rate does not change. For $\tau_{c}=0.75$ second, adhesion probability $\left(R_{a}\right)$ is 0.3 , i.e., approximately one third of the collisions resulted in successful adhesion. On the other hand, for $\tau_{c}=1.1, R_{a}=1$, i.e., all the collisions resulted in successful adhesion. Thus, $E\left[T_{d}\right]$ and $T_{\text {sys }}$ become constant after $\tau_{c}=1.1$ second.

\subsection{Effect of Number of Bonds Required for Suc- cessful Adhesion}

Another critical parameter defined in Section 3 was the number of bonds required for successful adhesion. The effect of number of bonds required for successful adhesion on MAMNET performance is analyzed in Fig. 11(c). With the increase in the number of bonds required for successful adhesion, the adhesion of nanomachines becomes more difficult. Therefore, the adhesion probability decreases, which implies a decrease in the interaction of nanomachines. Hence, average message delivery delay increases and system throughput decreases with increasing number of bonds required for successful adhesion.

\subsection{Effect of Molecular Neuro-Spike Communica- tion Parameters}

Here, the effects of molecular neuro-spike communication parameters on MAMNET performance are explored. Synaptic variability, threshold used for spike detection and signal-to-noise ratio on post-synaptic potential affect molecular neuro-spike communication and thus, MAMNET performance.

In Fig. 12(a), average message delivery delay is shown with varying $\theta$ for different $S N R$. The effect of $S N R$ on delay performance is obvious. With decreasing $S N R$, the transmission probability of information decreases and this yields higher delays. The effect of the threshold used for spike detection is more complicated. In Chapter 4, it is pointed out that the choice of the threshold for detection of spikes is very important for molecular neurospike communication. There exists a critical value for $\theta$ to achieve a high channel performance. In Fig. 12(a), the average message delivery delay decreases until a certain value of $\theta$. After that point, $E\left[T_{d}\right]$ starts to increase. This is because of the effect of $\theta$ on transmission probability. In Fig. 12(b), the effects of the threshold used for spike detection on transmission probability and average message delivery delay are shown. It can be seen that, there exists a critical value of $\theta$ which makes the transmission probability maximum.

Similar arguments follow for the average throughput and system throughput. The threshold value that makes the transmission probability maximum also maximizes the throughput. In Fig. 12(c) and 12(d), a reverse hook shape exists for the average throughput and system throughput with varying threshold. From Fig. 12(a), Fig. 12(c) and Fig. 12(d), we deduce that the critical value of threshold slightly changes with different $S N R$ values. Therefore, a threshold that goes well with all $S N R$ values should be chosen to achieve low message delivery delay and high system throughput.

\subsection{Effect of Number of Spike Bits Contained in a Message}

The number of spike bits used to encode a message is a critical parameter. Because of the limited capabilities of nanomachines, we do not expect them to generate a large amount of information. Nanomachines sensing the environment, generates information about the observed phenomena. The information generated by nanomachines are on the order of a few dozen of spike bits. The predefined message strings, and their length are determined before the deployment, and hence, nanomachines are accordingly programmed. The length of the messages depends on the diversity of the phenomena that is desired to be observed in the deployment environment, e.g., with 8 bits 256 different phenomena can be represented.

In Fig. 13(a), the performance of MAMNET is shown for varying $n$. With increasing $n$, the performance of MAMNET decreases. This is due to the decreased transmission probability of a message. Note that, the successful transmission probability of a message given in (24) exponentially decreases with increasing message length. This causes fewer nanomachines to get infected, and eventually, a higher message delivery delay. On the other hand, having more number of spike bits transmitted in a single message, one can expect a higher system throughput. However, the decrease in the successful message transmission probability overcomes the increase in the number of spike bits contained in a message. Therefore, system throughput decreases with increasing number of spike bits contained in a message.

\subsection{Effect of Number of Nanomachines}

The number of nanomachines in MAMNET is also a critical parameter for the performance MAMNET. In Fig. 13(b), average message delay and system throughput are shown for varying number of nanomachines. The increasing number of nanomachines increases the connectivity in MAMNET. Therefore, the message can be delivered to an infostation in a lower time. Furthermore, the system throughput increases with increasing number of nanomachines.

\section{Conclusion}

In this paper, using the stochastic models for collision and adhesion of nanomachines we first derive the rates for the interaction of nanomachines. Then, using the principles of neural communication we provide a new communication paradigm for the realization of mobile ad hoc molecular nanonetworks. We also investigate the performance of molecular neuro-spike channel model by examining single spike bit error probability and mutual information. Then we model the flow of a single message in MAMNET by 


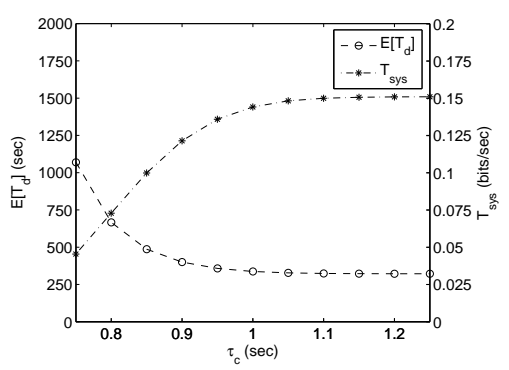

(a)

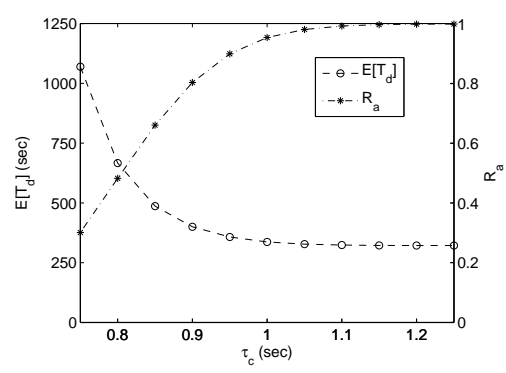

(b)

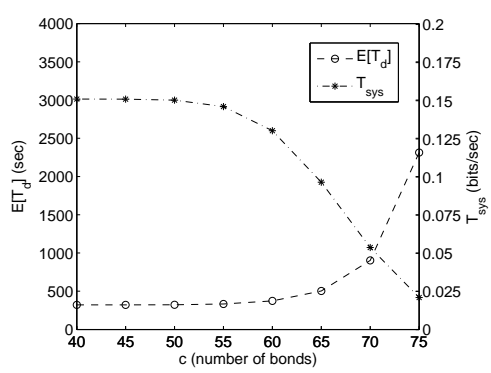

(c)

Fig. 11. (a) Average message delay and system throughput with varying contact time. (b) Adhesion probability and average message delay with varying contact time. (c) Average message delay with varying threshold value for different values of SNR.

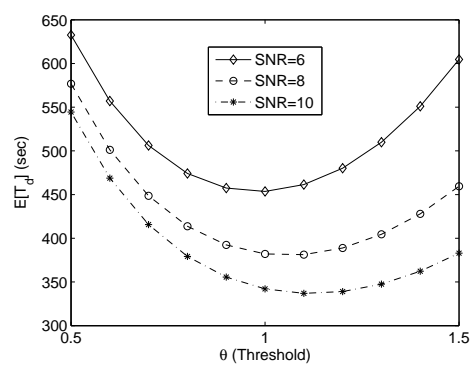

(a)

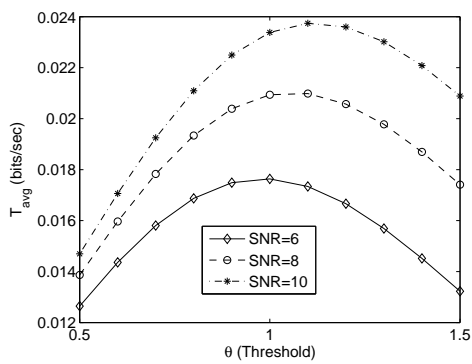

(c)

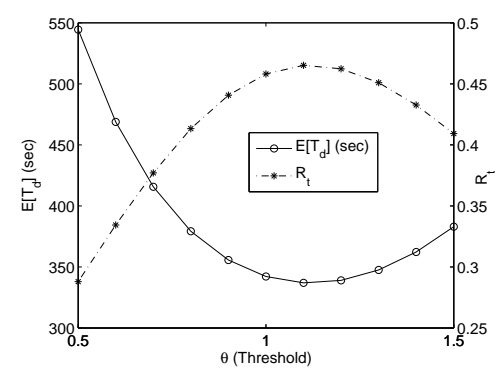

(b)

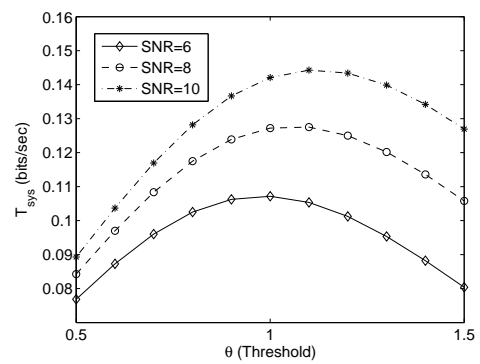

(d)

Fig. 12. (a) Average message delay (b) average message delay and transmission probability (c) average throughput (d) system throughput with varying threshold value for different values of SNR.

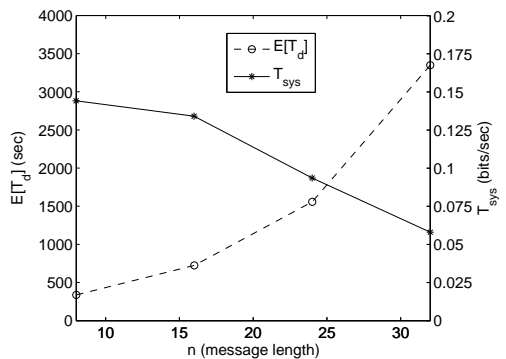

(a)

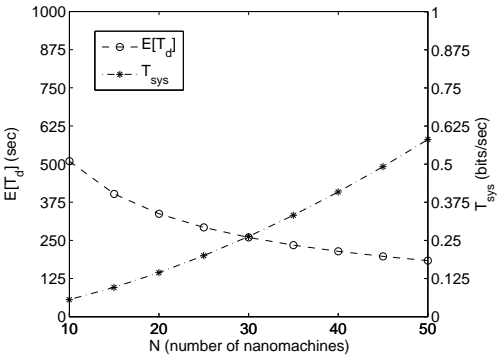

(b)

Fig. 13. Average message delay and system throughput with varying (a) number of spike bits contained in the message. (b) number of nanomachines.

using the principles of infectious disease spreading. We evaluate the performance of MAMNET based on average message delivery delay and system throughput expressions. Our models and numerical analysis clearly show that a mobile ad hoc nanonetwork can be realized with sufficiently low message delivery delay and sufficiently high system throughput. Numerical results also reveal that it is imperative to efficiently and effectively regulate the parameters to achieve better performance. 


\section{REFERENCES}

[1] I. F. Akyildiz, F. Brunetti, C. Blazquez, "NanoNetworking: A New Communication Paradigm", Computer Networks Journal (Elsevier), June 2008

[2] M. Pierobon, I. F. Akyildiz, "A Physical Channel Model for Molecular Communication in Nanonetworks", IEEE Journal on Selected Areas in Communications (J-SAC), Vol. 28, No. 4, May 2010, pp. 602-611.

[3] M, Gregori, and I. F. Akyildiz, "A New NanoNetwork Architecture using Flagellated Bacteria and Catalytic Nanomotors," IEEE Journal of Selected Areas in Communications (JSAC), Vol. 28, No. 4, pp. 612-619, May 2010.

[4] G. M. Whitesides, "The Once and Future Nano-machine", Scientific American, 2001

[5] S. Hiyama, Y. Moritani, T. Suda, R. Egashira, A. Enomoto, M. Moore and T. Nakano, "Molecular Communication", in Proc. NSTI Nanotech 2005, Anaheim, California, USA, 2005.

[6] B. Atakan, O. B. Akan, "On Channel Capacity and Error Compensation in Molecular Communication", Springer Trans. on Computational System Biology, vol. 10, 2008, pp. 59-80.

[7] M. Moore, A. Enomoto, T. Nakano, R. Egashira, T. Suda, A. Kayasuga, H. Kojima, H. Sakakibara, K. Oiwa, "A Design of a Molecular Communication System for Nanomachines Using Molecular Motors", in Proc. IEEE PERCOMW 2006, Italy, 2006.

[8] T. Nakano, T. Suda, M. Moore, R. Egashira, A. Enomoto, K, Arima, "Molecular Communication for Nanomachines Using Intercellular Calcium Signaling", in Proc. IEEE Conference on Nanotechnology 2005, Nagoya, Japan, July 2005.

[9] S. E. Chesla, P. Selveraj, C. Zu, "Measuring Two-Dimensional Receptor-Ligand Binding Kinetics by Micropipette", Biophysical Journal, vol. 75, no. 3, September, 1998, pp. 1553-1572.

[10] Y. Moritani, S. Hiyama, T. Suda, "Molecular Communication among Nanomachines Using Vesicles", in Proc. NSTI Nanotechnology Conference and Trade Show, 2006.

[11] B. Atakan, O. B. Akan, "Carbon nanotube-based nanoscale ad hoc networks", IEEE Communications Magazine, vol. 48, no. 6, 2010, pp. 129-135.

[12] J. Wiedermann, L. Petru, "Communicating Mobile Nano-Machines and Their Computational Power.", in Proc. ICST Conference on Nano-networks, Boston, USA, 2008.

[13] A. Grakoui, et. al., "The Immunological Synapse: A Molecular Machine Controlling T Cell Activation”, Science, vol. 285, no. 5425, 1999, pp. 221-227.

[14] R.A. Freitas, "Nanomedicine, Volume I: Basic Capabilities", Landes Biosience, 1999.

[15] D. A. McQuarrie, "Kinetics of small systems I", Chemical Physics, vol. 38, 1963, pp. 433-436.

[16] D. M. Blaugh, G. Resta, P. Santi, "A statistical analysis of the longrun node spatial distribution in mobile ad hoc networks", Wireless Networks, Vol 10, 2004, pp. 543-554.

[17] G. I. Bell, "Models for the specific adhesion of cells to cells", Science vol. 200. no. 4342, 12 May 1978, pp. 618-627.

[18] M. L. Dustin and D. R. Colman, "Neural and Immunological Synaptic Relations", Science vol. 298 no. 5594, 25 October 2002, pp. 785-789.

[19] R. L. Juliano, "Signal transduction by cell adhesion receptors and the cytoskeleton: functions of integrins, cadherins, selectins, and immunoglobulin-superfamily members", Annu. Rev. Pharmacol. Toxicol. vol. 42, 2002, pp. 283-323.

[20] A. Manwani, "Information-theoretic analysis of neuronal communication", Thesis(Phd), California Institute of Technology, 2000.

[21] L. J. DeFelice, "Introduction to membrane noise", Plenum Press, 1981.

[22] Z. J. Haas, T. Small, "A new networking model for biological applications of ad hoc sensor networks", IEEE/ACM Trans. on Networking, vol. 14, no 1, 2006, pp. 27-40.

[23] X. Zhang, G. Neglia, J. Kurose and D. Towsley, "Performance modeling of epidemic routing", Computer Networks, vol. 51, no. 10, 2007, pp. 2867-2891.

[24] A. Khelil, C. Becker, J. Tian, and K. Rothermel, "An epidemic model for information diffusion in MANETs", in Proc. 5th ACM International Workshop on Modeling Analysis and Simulation of Wireless and Mobile Systems, Atlanta, Georgia, September 2002.

[25] W. O. Kermack, A. G. McKendrick, "A contribution to the mathematical theory of epidemics", in Proc. R. Soc. Lond A, 115, 1927, pp. 700-721

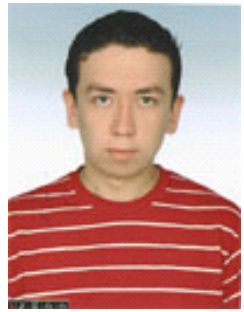

Aydin Guney received the B.Sc. and M.Sc degrees in electrical and electronics engineering from Middle East Technical University, Ankara, Turkey, in 2007 and 2010, respectively. He is currently pursuing his Ph.D degree at the Department of Electrical and Electronics Engineering, Middle Easy Technical University, and at the same time working as a test design engineer at Aselsan Inc. and his current research interests include molecular communication and

nanonetworks.

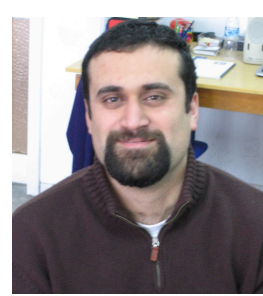

Baris Atakan (S'05) received the B.Sc. and M.Sc. degrees in electrical and electronics engineering from Ankara University and Middle East Technical University, Ankara, Turkey, in 2000 and 2005, respectively. He is currently a research assistant in the Next generation Wireless Communication Laboratory and pursuing his Ph.D degree at the Department of Electrical and Electronics Engineering, Koc University. His current research interests include biologically-inspired communication protocols for Wireless Sensor Networks and Cognitive Radio Networks, and nano-scale communication.

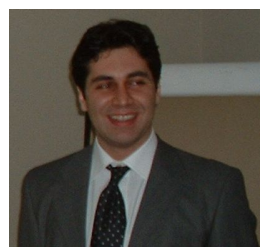

Ozgur B. Akan (M'00-SM'07) received the B.S. and M.S. degrees in electrical and electronics engineering from Bilkent University and Middle East Technical University, Ankara, Turkey, in 1999 and 2001, respectively. He received the Ph.D. degree in electrical and computer engineering from the Broadband and Wireless Networking Laboratory, School of Electrical and Computer Engineering, Georgia Institute of Technology, Atlanta, in 2004. He is currently Associate Professor with the Department of Electrical and Electronics Engineering, Koc University and the Director of Next-generation Wireless Communications Laboratory (NWCL). His current research interests are in wireless communications, bio-inspired communications, nanoscale and molecular communications, network information theory.

Dr. Akan is an Associate Editor for IEEE Transactions on Vehicular Technology, International Journal of Communication Systems (Wiley), Nano Communication Networks Journal (Elsevier). He served as an Editor for ACM/Springer Wireless Networks (WINET) Journal (2004-2010), as an Area Editor for AD HOC Networks Journal (Elsevier) (2004-2008), as a Guest Editor for several special issues, as the TPC Co-Chair for the 5th ICST Conference on Nano Networks, as the TPC Co-Chair for the 13th ACM International Conference on Modeling, Analysis and Simulation of Wireless and Mobile Systems (ACM MSWiM 2010), the General Co-Chair for The Third International Conference on Bio-Inspired Models of Network, Information, and Computing Systems (ICST/IEEE BIONETICS 2008), the European Vice Chair for The Second International Conference on NanoNetworks (ICST/ACM Nano-Net 2007), an International Vice Chair for IEEE INFOCOM 2006, and in organizing committees and technical program committees of many other international conferences. $\mathrm{He}$ is the Vice President for IEEE Communications Society - Turkey Section. $\mathrm{He}$ is an IEEE Senior Member (Communications Society), and a member of ACM. Dr. Akan received the IBM Faculty Award twice in 2010 and 2008, Turkish Academy of Sciences Distinguished Young Scientist Award 2008 (TUBA-GEBIP). 